\title{
New Black Hole Solutions in Brans-Dicke Theory of Gravity
}

\author{
Hongsu Kim* \\ Department of Astronomy and Atmospheric Sciences \\ Kyungpook National University, Taegu, 702-701, KOREA
}

(October, 1998)

\begin{abstract}
Existence check of non-trivial, stationary axisymmetric black hole solutions in Brans-Dicke theory of gravity in different direction from those of Penrose, Thorne and Dykla, and Hawking is performed. Namely, working directly with the known explicit spacetime solutions in Brans-Dicke theory, it is found that non-trivial Kerr-Newman-type black hole solutions different from general relativistic solutions could occur for the generic Brans-Dicke parameter values $-5 / 2 \leq \omega<-3 / 2$. Finally, issues like whether these new black holes carry scalar hair and can really arise in nature and if they can, what the associated physical implications would be are discussed carefully.
\end{abstract}

PACS numbers: 04.20.Jb, 04.50.+h, 04.70.-s

*e-mail : hongsu@vega.kyungpook.ac.kr 


\section{Introduction}

Of all the alternative theories of classical gravity to Einstein's general relativity, perhaps the Brans-Dicke (BD) theory [1] is the most studied and hence the best-known. This theory can be thought of as a minimal extension of general relativity designed to properly accomodate both Mach's principle [2] and Dirac's large number hypothesis [2]. Namely, the theory employs the viewpoint in which the Newton's constant $G$ is allowed to vary with space and time and can be written in terms of a scalar ("BD scalar") field as $G=1 / \Phi$. In this work, we are interested in the existence of exact solutions to the BD field equations that can describe rotating, charged black hole spacetimes and their detailed structure. And if there are, we would like to know whether they are non-trivial ones different from general relativistic black hole solutions. As is well-known, even in Einstein's general relativity, to find the exact solutions to the highly non-linear Einstein field equations is a formidable task. For this reason, algorithms generating exact, new solutions from the known solutions of simpler situations have been actively looked for and actually quite a few were found. In BD theory of gravity, the field equations are even more complex and thus it is natural to seek similar algorithms generating exact solutions from the already known simpler solutions either of the BD theory or of the conventional Einstein gravity. To the best of our knowledge, methods thus far discovered along this line includes those of Janis et al., Buchdahl, McIntosh, Tupper, Tiwari and Nayak, and Singh and Rai [3]. In particular, Tiwari and Nayak [3] proposed an algorithm that allows us to generate stationary, axisymmetric solutions in vacuum BD theory from the known Kerr solution [6] in vacuum Einstein theory and later on Singh and Rai [3] generalized this method to the one that generates stationary, axisymmetric, charged solutions in BD-Maxwell theory from the known Kerr-Newman (KN) solution [6] in Einstein-Maxwell theory. Thus in the present work, we shall take, as the Kerr-Newman-type

solutions in BD-Maxwell theory (henceforth "BDKN" solutions), the ones constructed by Singh and Rai to explore if it can descibe non-trivial black hole spacetimes different from those described by the standard KN solution in Einstein-Maxwell theory.

\section{Non-trivial BDKN black hole solutions}


We begin by briefly reviewing the algorithm proposed first by Tiwari and Nayak and generalized later by Singh and Rai. Consider the BD-Maxwell theory described by the action

$$
S=\int d^{4} x \sqrt{g}\left[\frac{1}{16 \pi}\left(\Phi R-\omega \frac{\nabla_{\alpha} \Phi \nabla^{\alpha} \Phi}{\Phi}\right)-\frac{1}{4} F_{\alpha \beta} F^{\alpha \beta}\right]
$$

where $\Phi$ is the BD scalar field and $\omega$ is the generic parameter of the theory. Extremizing this action then with respect to the metric $g_{\mu \nu}$, the BD scalar field $\Phi$, and the Maxwell gauge field $A_{\mu}$ (with the field strength $F_{\mu \nu}=\nabla_{\mu} A_{\nu}-\nabla_{\nu} A_{\mu}$ ) yields the classical field equations given respectively by

$$
G_{\mu \nu}=R_{\mu \nu}-\frac{1}{2} g_{\mu \nu} R=\frac{8 \pi}{\Phi} T_{\mu \nu}^{M}+8 \pi T_{\mu \nu}^{B D},
$$

where

$$
\begin{aligned}
T_{\mu \nu}^{M} & =F_{\mu \alpha} F_{\nu}^{\alpha}-\frac{1}{4} g_{\mu \nu} F_{\alpha \beta} F^{\alpha \beta}, \\
T_{\mu \nu}^{B D} & =\frac{1}{8 \pi}\left[\frac{\omega}{\Phi^{2}}\left(\nabla_{\mu} \Phi \nabla_{\nu} \Phi-\frac{1}{2} g_{\mu \nu} \nabla_{\alpha} \Phi \nabla^{\alpha} \Phi\right)+\frac{1}{\Phi}\left(\nabla_{\mu} \nabla_{\nu} \Phi-g_{\mu \nu} \nabla_{\alpha} \nabla^{\alpha} \Phi\right)\right] \\
\text { and } & \\
\nabla_{\alpha} \nabla^{\alpha} \Phi & =\frac{8 \pi}{(2 \omega+3)} T_{\lambda}^{M \lambda}=0, \quad \nabla_{\mu} F^{\mu \nu}=0, \quad \nabla_{\mu} \tilde{F}^{\mu \nu}=0
\end{aligned}
$$

with the last equation being the Bianchi identity and $\tilde{F}_{\mu \nu}=\frac{1}{2} \epsilon_{\mu \nu}^{\alpha \beta} F_{\alpha \beta}$. And the EinsteinMaxwell theory is the $\omega \rightarrow \infty$ limit of this BD-Maxwell theory. Note that in the action and hence in the classical field equations, there are no direct interactions between the BD scalar field $\Phi$ and the ordinary matter, i.e., the Maxwell gauge field $A_{\mu}$. Indeed this is the essential feature of the BD scalar field $\Phi$ that distinguishes it from "dilaton" fields in other scalar-tensor theories such as Kaluza-Klein theories or low-energy effective string theories where the dilaton-matter couplings generically occur as a result of dimensional reduction. (Here we would like to stress that we shall work in the context of original BD theory format not some conformal transformation of it.) As a matter of fact, it is the original spirit [1] of BD theory of gravity in which the BD scalar field $\Phi$ is prescribed to remain strictly massless by forbidding its direct interaction with matter fields. Now the algorithm of Tiwari and Nayak, and Singh and Rai goes as follows. Let the metric for a stationary, axisymmetric, charged solution to Einstein-Maxwell field equations take the form 


$$
d s^{2}=-e^{2 U_{E}}\left(d t+W_{E} d \phi\right)^{2}+e^{2\left(k_{E}-U_{E}\right)}\left[\left(d x^{1}\right)^{2}+\left(d x^{2}\right)^{2}\right]+h_{E}^{2} e^{-2 U_{E}} d \phi^{2}
$$

while the metric for a stationary, axisymmetric, charged solution to BD-Maxwell field equations be

$$
d s^{2}=-e^{2 U_{B D}}\left(d t+W_{B D} d \phi\right)^{2}+e^{2\left(k_{B D}-U_{B D}\right)}\left[\left(d x^{1}\right)^{2}+\left(d x^{2}\right)^{2}\right]+h_{B D}^{2} e^{-2 U_{B D}} d \phi^{2}
$$

where $U, W, k$ and $h$ are functions of $x^{1}$ and $x^{2}$ only. The significance of the choice of the metric in this form has been thoroughly discussed by Matzner and Misner [4] and Misra and Pandey [5]. Tiwari and Nayak, and Singh and Rai first wrote down the Einstein-Maxwell and BD-Maxwell field equations for the choice of metrics in eq.(3) and (4) respectively. Comparing the two sets of field equations closely, they realized that stationary, axisymmetric solutions of the BD-Maxwell field equations are obtainable from those of Einstein-Maxwell field equations provided certain relations between metric functions hold.

That is, if $\left(W_{E}, k_{E}, U_{E}, h_{E}, A_{\mu}^{E}\right)$ form a stationary, axisymmetric solution to the EinsteinMaxwell field equations for the metric in eq.(3), then a corresponding stationary, axisymmetric solution to the BD-Maxwell field equations for the metric in eq.(4) is given by $\left(W_{B D}, k_{B D}, U_{B D}, h_{B D}, A_{\mu}^{B D}\right)$ where

$$
\begin{aligned}
W_{B D} & =W_{E}, \quad k_{B D}=k_{E}, \quad U_{B D}=U_{E}-\frac{1}{2} \log \Phi \\
h_{B D} & =\left[h_{E}\right]^{(2 \omega-1) /(2 \omega+3)}, \quad \Phi=\left[h_{E}\right]^{4 /(2 \omega+3)}, \quad A_{\mu}^{B D}=A_{\mu}^{E} .
\end{aligned}
$$

Now what remains is to apply this method to obtain the Kerr-Newman-type solution in BD-Maxwell theory (BDKN solution) from the known Kerr-Newman (KN) solution [6] in Einstein-Maxwell theory. And to do so, one needs some preparation which involves casting the $\mathrm{KN}$ solution given in Boyer-Lindquist coordinates [7] $(t, r, \theta, \phi)$ in the metric form in eq.(3) by performing a coordinate transformation (of $r$ alone) suggested by Misra and Pandey [5]. Namely, we start with the KN solution written in Boyer-Lindquist coordinates

$$
\begin{aligned}
d s^{2} & =-d t^{2}+\Sigma\left(d \theta^{2}+\frac{d r^{2}}{\Delta}\right)+\left(r^{2}+a^{2}\right) \sin ^{2} \theta d \phi^{2}+\frac{\left(2 M r-e^{2}\right)}{\Sigma}\left[d t-a \sin ^{2} \theta d \phi\right]^{2}, \\
A_{\mu} & =-\frac{e r}{\Sigma}\left[\delta_{\mu}^{t}-a \sin ^{2} \theta \delta_{\mu}^{\phi}\right]
\end{aligned}
$$


where $\Sigma=r^{2}+a^{2} \cos ^{2} \theta$ and $\Delta=r^{2}-2 M r+a^{2}+e^{2}$ with $M, a$, and $e$ denoting the ADM mass, angular momentum per unit mass, and the electric charge respectively. Consider now the transformation of the radial coordinate introduced by Misra and Pandey [5]

$$
r=e^{R}+M+\frac{\left(M^{2}-a^{2}-e^{2}\right)}{4} e^{-R}
$$

which gives $d r^{2} / \Delta=d R^{2}$. Then the KN solution can now be cast in the form in eq.(3), i.e.,

$$
d s^{2}=-\left[\frac{\Delta-a^{2} \sin ^{2} \theta}{\Sigma}\right](d t+W d \phi)^{2}+\Sigma\left[d \theta^{2}+d R^{2}+\frac{\Delta \sin ^{2} \theta}{\Delta-a^{2} \sin ^{2} \theta} d \phi^{2}\right]
$$

with now $\Sigma=L^{2}+a^{2} \cos ^{2} \theta$ and $\Delta=L^{2}-2 M L+a^{2}+e^{2}$ where we set, as a short-hand notation, $L \equiv e^{R}+M+\frac{\left(M^{2}-a^{2}-e^{2}\right)}{4} e^{-R}$. Now we can read off the metric components as

$$
\begin{aligned}
& e^{2 U_{E}}=\left[\frac{\Delta-a^{2} \sin ^{2} \theta}{\Sigma}\right], \quad W_{E}=\frac{a \sin ^{2} \theta\left(L^{2}+a^{2}-\Delta\right)}{\Delta-a^{2} \sin ^{2} \theta}, \\
& e^{2 k_{E}}=\left(\Delta-a^{2} \sin ^{2} \theta\right), \quad h_{E}^{2}=\Delta \sin ^{2} \theta \text {. }
\end{aligned}
$$

Then using the rule in eq.(5) in the algorithm by Tiwari and Nayak, and Singh and Rai, we can now construct BDKN solution in BD-Maxwell theory as

$$
\begin{aligned}
d s^{2} & =-\left(\frac{L^{2}+a^{2} \cos ^{2} \theta-2 M L+e^{2}}{L^{2}+a^{2} \cos ^{2} \theta}\right)\left(L^{2}+a^{2}-2 M L+e^{2}\right)^{-2 /(2 \omega+3)} \sin ^{-4 /(2 \omega+3)} \theta \\
& \times\left[d t+\frac{a \sin ^{2} \theta\left(2 M L-e^{2}\right)}{L^{2}+a^{2} \cos ^{2} \theta-2 M L+e^{2}} d \phi\right]^{2} \\
& +\left(L^{2}+a^{2}-2 M L+e^{2}\right)^{2 /(2 \omega+3)} \sin ^{4 /(2 \omega+3)} \theta\left(L^{2}+a^{2} \cos ^{2} \theta\right)\left[d \theta^{2}+d R^{2}\right] \\
& +\left(L^{2}+a^{2}-2 M L+e^{2}\right)^{(2 \omega+1) /(2 \omega+3)} \sin ^{2(2 \omega+1) /(2 \omega+3)} \theta\left(\frac{L^{2}+a^{2} \cos ^{2} \theta}{L^{2}+a^{2} \cos ^{2} \theta-2 M L+e^{2}}\right) d \phi^{2}, \\
\Phi(R, \theta) & =\left(L^{2}+a^{2}-2 M L+e^{2}\right)^{2 /(2 \omega+3)} \sin ^{4 /(2 \omega+3)} \theta, \\
A_{\mu} & =-\frac{e L}{L^{2}+a^{2} \cos ^{2} \theta}\left[\delta_{\mu}^{t}-a \sin ^{2} \theta \delta_{\mu}^{\phi}\right] .
\end{aligned}
$$

Then by transforming back to the standard Boyer-Lindquist coordinates using eq.(7), we finally arrive at the BDKN solution in Boyer-Lindquist coordinates given by

$$
\begin{aligned}
d s^{2} & =\Delta^{-2 /(2 \omega+3)} \sin ^{-4 /(2 \omega+3)} \theta\left[-\left(\frac{\Delta-a^{2} \sin ^{2} \theta}{\Sigma}\right) d t^{2}-\frac{2 a \sin ^{2} \theta\left(r^{2}+a^{2}-\Delta\right)}{\Sigma} d t d \phi\right. \\
& \left.+\left(\frac{\left(r^{2}+a^{2}\right)^{2}-\Delta a^{2} \sin ^{2} \theta}{\Sigma}\right) \sin ^{2} \theta d \phi^{2}\right]+\Delta^{2 /(2 \omega+3)} \sin ^{4 /(2 \omega+3)} \theta\left[\frac{\Sigma}{\Delta} d r^{2}+\Sigma d \theta^{2}\right], \\
\Phi(r, \theta) & =\Delta^{2 /(2 \omega+3)} \sin ^{4 /(2 \omega+3)} \theta, \quad A_{\mu}=-\frac{e r}{\Sigma}\left[\delta_{\mu}^{t}-a \sin ^{2} \theta \delta_{\mu}^{\phi}\right] .
\end{aligned}
$$


Note that as $\omega \rightarrow \infty$, this BDKN solution goes over to the standard KN solution as it should since the $\omega \rightarrow \infty$ limit of BD theory is the Einstein gravity. Now that we have an exact, electrovac, stationary axisymmetric solution to the BD-Maxwell theory. Then it is natural to ask if this BDKN solution can describe a black hole spacetime resulted from a gravitational collapse. And if it can, furthermore one might be curious whether this BDKN solution or its three special cases (i.e., BD-Schwarzschild, BD-Reissner-Nordstrom or BD-Kerr solutions) could describe non-trivial black hole spacetimes which are different from those described by their general relativistic counterparts. Indeed, questions of this sort had been raised long ago, and actually Penrose [8] conjectured that even in BD theory of gravity, the relativistic gravitational collapse in three spatial dimensions would produce black holes identical to those in general relativity. And this conjecture received some support from the work of Thorne and Dykla [8] in which they presented four pieces of evidence in favor of the conjecture by employing mainly the "large- $\omega$ " expansion scheme (recall that in the limit $\omega \rightarrow \infty$, the BD theory goes over to the general relativity). As Thorne and Dykla mentioned in their work, however, the conjecture of Penrose was not fully proved since detailed analysis of the collapse with arbitrary, finite values of the generic BD parameter $\omega$ is needed. In this regard, we now seem to be in a better shape toward the serious investigation on the validity of the conjecture since we have an exact, stationary axisymmetric solution possessing arbitrary $\omega$ values which was not available at the time. Therefore we begin with the bottomline qualification for the BDKN solution in BD-Maxwell theory to describe a rotating, charged black hole spacetime, namely the possible occurrence of non-singular event horizon. Then along this line, perhaps the most natural first step is to ask under what circumstances the Killing horizons develop. Just like the KN solution in Einstein-Maxwell theory, this BDKN solution is stationary and axisymmetric and hence possesses the time translational Killing field $\xi^{\mu}=(\partial / \partial t)^{\mu}$ and the rotational Killing field $\psi^{\mu}=(\partial / \partial \phi)^{\mu}$ correspondingly and it is their linear combination, $\chi^{\mu}=\xi^{\mu}+\Omega_{H} \psi^{\mu}$ which is normal to the Killing horizons, if any (here $\Omega_{H}$ denotes the angular velocity of the Killing horizon). And if Killing horizons are present, they occur at points where $\chi^{\mu}$ becomes null which turn out to be zeroes of $\Delta^{(2 \omega+1) /(2 \omega+3)}=0$. 
Thus first for $\omega=-1 / 2$, obviously no horizon occurs. Next for $\left(\frac{2 \omega+1}{2 \omega+3}\right)>0$, i.e., for $\omega<-3 / 2$ or $\omega>-1 / 2$, two Killing horizons occur at $r_{ \pm}=M \pm\left(M^{2}-a^{2}-e^{2}\right)^{1 / 2}$ (provided $\left.M^{2} \geq a^{2}+e^{2}\right)$ which are precisely the same locations as those of Killing horizons of KN black holes in Einstein-Maxwell theory. Now since the formation of horizons appears to be possible, next we investigate their nature. And to this end, we examine behaviors of the invariant curvature polynomials such as $R, R_{\mu \nu} R^{\mu \nu}$ and $R_{\mu \nu \alpha \beta} R^{\mu \nu \alpha \beta}$, the surface gravity $\kappa$ and the energy density of the BD scalar field $T_{\mu \nu}^{B D} \xi^{\mu} \xi^{\nu}$ on these candidates for Killing horizons. And as we mentioned above, since the bottomline qualification for the black hole interpretation of BDKN solution is the regularity of the horizon candidate, we begin with the examination of behavior of invariant curvature polynomials on the horizon candidate at which $\Delta=0$. First, the curvature scalar is calculated to be

$$
\begin{aligned}
R & =\frac{\omega}{\Phi^{2}} g^{\alpha \beta} \nabla_{\alpha} \Phi \nabla_{\beta} \Phi+\frac{3}{\Phi} \nabla_{\alpha} \nabla^{\alpha} \Phi \\
& =\omega\left(\frac{4}{2 \omega+3}\right)^{2} \frac{1}{\Sigma} \sin ^{-4 /(2 \omega+3)} \theta\left[(r-M)^{2} \Delta^{-(2 \omega+5) /(2 \omega+3)}+\cot ^{2} \theta \Delta^{-2 /(2 \omega+3)}\right] .
\end{aligned}
$$

As was the case with $\mathrm{KN}$ black hole solutions, it also blows up at $\Sigma=0$ (i.e., $r=0, \theta=\pi / 2$ ) indicating that the BDKN black hole solution also has the curvature singularity with the same "ring" structure. The direct computation of the other two curvature polynomials, i.e., the Ricci square $R_{\mu \nu} R^{\mu \nu}$ and the Kretschmann curvature invariant $R_{\mu \nu \alpha \beta} R^{\mu \nu \alpha \beta}$ for this BDKN solution is a formidable job. But a close inspection reveals that indeed we can save considerable amount of labor. Namely, consider now the Brans-Dicke-Schwarzschild (BDS) spacetime solution that can be obtained by setting $a=e=0$ in the BDKN solution in eq. (11)

$$
\begin{aligned}
d s^{2} & =\Delta^{-2 /(2 \omega+3)} \sin ^{-4 /(2 \omega+3)} \theta\left[-\left(1-\frac{2 M}{r}\right) d t^{2}+r^{2} \sin ^{2} \theta d \phi^{2}\right] \\
& +\Delta^{2 /(2 \omega+3)} \sin ^{4 /(2 \omega+3)} \theta\left[\left(1-\frac{2 M}{r}\right)^{-1} d r^{2}+r^{2} d \theta^{2}\right], \\
& \Phi(r, \theta)=\Delta^{2 /(2 \omega+3)} \sin ^{4 /(2 \omega+3)} \theta
\end{aligned}
$$

where now $\Delta=r(r-2 M)$. A remarkable feature of this BDS solution is the fact that, unlike the Schwarzschild solution in general relativity, the spacetime it describes is static (i.e., non- 
rotating) but not spherically-symmetric. Thus computing $R_{\mu \nu} R^{\mu \nu}$ and $R_{\mu \nu \alpha \beta} R^{\mu \nu \alpha \beta}$ for this BDS solution and examining their behaviors on the horizon candidate at which $\Delta=0$ would be sufficient to envisage the possibility of regular event horizon for both BDS and BDKN solutions. In addition, another noticeable characteristic of both BDKN and BDS spacetime solutions is that they have possible coordinate singularities not only at the outer event horizon where $\Delta=0$ but also along the symmetry axis $\theta=0, \pi$. Thus in order to explore the nature of this metric singularity along the symmetry axis, the computation of invariant curvature polynomials looks necessary. The result of the computation of the Ricci square and the Kretschmann curvature invariant for this BDS solution is given in the appendix. And it is a straightforward matter to realize that the two invariant curvature polynomials $R_{\mu \nu} R^{\mu \nu}$ and $R_{\mu \nu \alpha \beta} R^{\mu \nu \alpha \beta}$ given in the appendix and the curvature scalar $R$ given above in eq.(12) become finite both on the horizon candidate at which $\Delta=0$ and along the symmetry axis $\theta=0, \pi$ provided the generic $\mathrm{BD} \omega$-parameter takes values in the range $-5 / 2 \leq \omega<-3 / 2$. Next, we turn to the computation of surface gravity at the Killing horizons, $\kappa_{ \pm}$. Generally, the surface gravity $\kappa$ is defined in a gravity theory- independent manner as follows. Since the horizon is a null surface, there we have $\chi^{\mu} \chi_{\mu}=0$ where $\chi^{\mu}$ is the Killing field normal to the horizon we defined above. This implies that $\nabla^{\mu}\left(\chi^{\nu} \chi_{\nu}\right)$ is also normal to the horizon. Thus on the horizon, there exists a function $\kappa$ such that

$$
\nabla^{\mu}\left(\chi^{\nu} \chi_{\nu}\right)=-2 \kappa \chi^{\mu} \quad \text { or } \quad \chi^{\nu} \nabla_{\nu} \chi_{\mu}=\kappa \chi_{\mu}
$$

from which it can be derived that

$$
\kappa^{2}=-\frac{1}{2}\left(\nabla^{\mu} \chi^{\nu}\right)\left(\nabla_{\mu} \chi_{\nu}\right)
$$

where the evaluation on the horizon is understood. Now, for the non-trivial BDKN black hole solution at hand, a straightforward albeit somewhat tedious calculation yields

$$
\kappa_{ \pm}=\Delta^{-2 /(2 \omega+3)}\left(r_{ \pm}\right) \sin ^{-4 /(2 \omega+3)} \theta\left(\frac{2 \omega+1}{2 \omega+3}\right) \frac{\left(r_{ \pm}-r_{\mp}\right)}{2\left(r_{ \pm}^{2}+a^{2}\right)}
$$

Lastly, the energy density of the BD scalar field is computed using eq.(2) as 


$$
\begin{aligned}
& T_{\mu \nu}^{B D} \xi^{\mu} \xi^{\nu}=T_{t t}^{B D} \\
& =\frac{1}{8 \pi} \Delta^{-4 /(2 \omega+3)} \sin ^{-8 /(2 \omega+3)} \theta\left[\frac{\omega}{2}\left(\frac{4}{2 \omega+3}\right)^{2}\left(\frac{(r-M)^{2}}{\Delta}+\cot ^{2} \theta\right)\left(\frac{\Delta-a^{2} \sin ^{2} \theta}{\Sigma^{2}}\right)\right. \\
& +\left(\frac{4}{2 \omega+3}\right) \frac{1}{\Sigma^{2}}\left\{\cot ^{2} \theta\left(\left(\frac{2 \omega+1}{2 \omega+3}\right) a^{2} \sin ^{2} \theta+\left(\frac{2}{2 \omega+3}\right) \Delta-a^{2} \sin ^{2} \theta\left(\frac{\Delta-a^{2} \sin ^{2} \theta}{\Sigma}\right)\right)\right. \\
& \left.\left.-\left(\frac{r-M}{\Delta}\right)\left(\left(\frac{2 \omega+1}{2 \omega+3}\right)(r-M) \Delta+\left(\frac{2}{2 \omega+3}\right)(r-M) a^{2} \sin ^{2} \theta-r \Delta\left(\frac{\Delta-a^{2} \sin ^{2} \theta}{\Sigma}\right)\right)\right\}\right] .
\end{aligned}
$$

It is interesting to note that this energy density of the BD scalar field also blows up at the curvature singularity $\Sigma=0$.

Now (i) for $\omega \rightarrow \infty, R=0, \kappa_{ \pm}=\left(r_{ \pm}-r_{\mp}\right) / 2\left(r_{ \pm}^{2}+a^{2}\right)$ and $T_{\mu \nu}^{B D} \xi^{\mu} \xi^{\nu}=0$ on the surfaces $r=r_{ \pm}$. This is an anticipated result since this is the correct KN black hole limit in Einstein-Maxwell theory. (ii) Next for $\infty>\omega>-1 / 2$, on the surfaces $r=r_{ \pm}$, $\left(R, R_{\mu \nu} R^{\mu \nu}, R_{\mu \nu \alpha \beta} R^{\mu \nu \alpha \beta}\right) \rightarrow \infty, \kappa_{ \pm} \rightarrow \infty$ and $T_{\mu \nu}^{B D} \xi^{\mu} \xi^{\nu} \rightarrow \infty$ with $\Phi\left(r_{ \pm}, \theta\right)=0$. This indicates that the surfaces $r=r_{ \pm}$are singular and fail to act as horizons and hence the corresponding metric cannot describe a black hole spacetime. (iii) Finally for $\omega<-3 / 2$, or more precisely for $-5 / 2 \leq \omega<-3 / 2$, on the surfaces $r=r_{ \pm},\left(R, R_{\mu \nu} R^{\mu \nu}, R_{\mu \nu \alpha \beta} R^{\mu \nu \alpha \beta}\right)=0$ (or const. particularly for $\omega=-5 / 2), \kappa_{ \pm}=0$ and $T_{\mu \nu}^{B D} \xi^{\mu} \xi^{\nu}=0$ with $\Phi\left(r_{ \pm}, \theta\right) \rightarrow \infty$. Namely the curvature invariants are finite, surface gravity is zero and the BD scalar field satisfies the weak energy condition although its value diverges there. (Here, infinite value of $\Phi$ indicates that the effective Newton's constant tends to zero.) Thus in this range of the $\omega$-values, the surfaces $r=r_{ \pm}$may act as regular Killing horizons and hence the corresponding BDKN metric solution appears to describe non-trivial black hole spacetimes different from those in Einstein-Maxwell theory. In particular for $\omega=-5 / 2$, the corresponding non-trivial BDKN black hole solution singles out with a relatively simple form given by

$$
\begin{aligned}
d s^{2} & =-\left[\frac{\Delta-a^{2} \sin ^{2} \theta}{\Sigma}\right] \Delta \sin ^{2} \theta d t^{2}-\frac{2 a \sin ^{4} \theta\left(r^{2}+a^{2}-\Delta\right) \Delta}{\Sigma} d t d \phi \\
& +\left[\frac{\left(r^{2}+a^{2}\right)^{2}-\Delta a^{2} \sin ^{2} \theta}{\Sigma}\right] \Delta \sin ^{4} \theta d \phi^{2}+\frac{\Sigma}{\Delta^{2} \sin ^{2} \theta} d r^{2}+\frac{\Sigma}{\Delta \sin ^{2} \theta} d \theta^{2}, \\
\Phi(r, \theta) & =\frac{1}{\Delta \sin ^{2} \theta} .
\end{aligned}
$$

Lastly, for the rest of the $\omega$-values, i.e., for $\omega<-5 / 2$, the surfaces $r=r_{ \pm}$are singular 
horizons on which $\left(R, R_{\mu \nu} R^{\mu \nu}, R_{\mu \nu \alpha \beta} R^{\mu \nu \alpha \beta}\right) \rightarrow \infty, \kappa_{+}=0$ and $T_{\mu \nu}^{B D} \xi^{\mu} \xi^{\nu}=0$ and for $-3 / 2<\omega \leq-1 / 2$, no Killing horizon develops and thus the curvature singularity at $\Sigma=0$ is naked. Therefore it now appears that for the values of the generic BD $\omega$-parameter in the limited range $-5 / 2 \leq \omega<-3 / 2$, the BDKN solution in BD-Maxwell theory may describe non-trivial black hole spacetimes.

\section{Nature of BDKN black holes}

Now that we have non-trivial BDKN black hole solutions. It seems then natural to explore its thermodynamics and causal structure in some more detail. Firstly, these BDKN black hole solutions have vanishing surface gravity at the event horizon, $\kappa_{+}=0$ and hence zero Hawking temperature, $T_{H}=\kappa_{+} / 2 \pi=0$. In other words, they do not radiate and hence are completely "dark and cold". Certainly, this is a very bizzare feature in sharp contrast to evaporating black holes in general relativity. Next, we turn to their causal structure. As noted earlier, the two Killing horizons, i.e., the outer event horizon and the inner Cauchy horizon turn out to occur precisely at the same locations (i.e., same coordinate distances) as those of KN black hole solutions in Einstein-Maxwell theory, i.e., at $r_{ \pm}=M \pm\left(M^{2}-a^{2}-e^{2}\right)^{1 / 2}$. Also it is interesting to note that the proper area of the event horizon at $r=r_{+}$,

$$
A=\int_{r_{+}} d \theta d \phi\left(g_{\theta \theta} g_{\phi \phi}\right)^{1 / 2}=4 \pi\left(r_{+}^{2}+a^{2}\right)
$$

is again exactly the same as that of standard KN black hole spacetime. In addition, its angular velocity at the event horizon coincides with that of standard KN solution as well

$$
-W_{B D}^{-1}\left(r_{+}\right)=\frac{a}{r_{+}^{2}+a^{2}}=-W_{E}^{-1}\left(r_{+}\right)
$$

Next, observe that the norm of the time translational Killing field

$$
\xi^{\mu} \xi_{\mu}=g_{t t}=-\Delta^{-2 /(2 \omega+3)} \sin ^{-4 /(2 \omega+3)} \theta\left[\frac{\Delta-a^{2} \sin ^{2} \theta}{\Sigma}\right]
$$

goes like negative $\left(r_{-}<r<r_{+}\right) \rightarrow$ positive $\left(r_{+}<r<r_{s}\right) \rightarrow$ negative $\left(r>r_{s}\right)$ with $r_{s}=M+\left(M^{2}-a^{2} \cos ^{2} \theta-e^{2}\right)^{1 / 2}>r_{+}$being the larger root of $\xi^{\mu} \xi_{\mu}$, indicating that $\xi^{\mu}$ behaves as timelike $\rightarrow$ spacelike $\rightarrow$ timelike correspondingly. And particularly the region in 
which $\xi^{\mu}$ stays spacelike extends outside hole's event horizon. This region is the so-called "ergoregion" and its outer boundary on which $\xi^{\mu}$ becomes null, i.e., $r=r_{s}$ is called "static limit" since inside of which no observer can possibly remain static. Thus if we recall the location of the static limit in standard KN black hole solution, we can realize that even the locations of ergoregions in two black hole spacetimes, $\mathrm{KN}$ and $\mathrm{BDKN}$, are the same as well. Namely in two theories, i.e., the BD-Maxwell theory and the Einstein-Maxwell theory, rotating, charged black hole solutions turn out to possess identical causal structure (i.e., the locations of ring singularities, two Killing horizons and static limits are the same) and hence exhibit the same global topology. Thus actually what distinguishes the BDKN black hole spacetime from its general relativity's counterpart, i.e., the KN black hole is the local geometry alone such as the curvature characterized by the specific $\omega$-values, $-5 / 2 \leq \omega<-3 / 2$. At this point, perhaps it is relevant to mention the behavior of the BD scalar field which plays unique role only in BD theory of gravity. Independently of Penrose [8] and of Thorne and Dykla [8], Hawking [9] also explored the possible existence of black hole solutions in BD theory and put forward a theorem which states that stationary black holes in BD theory are identical to those in general relativity. To be a little more concrete, Hawking extended some of his theorems for general relativistic black holes to BD theory and showed that any object collapsing to a black hole in BD theory must settle into final equilibrium state which is either Schwarzschild or Kerr spacetime. And in doing so, he "assumed" that the BD scalar field $\Phi$ satisfies the weak energy condition and is constant outside the black hole. Therefore now one may be puzzled as we realized in the present work that non-trivial BDKN black hole solutions different from general relativistic KN solution could exist in seemingly contradiction to Hawking's theorem. There is, however, no contradiction. Hawking deduced the theorem by manipulating the BD field equations and most crucially "assuming" the strict conditions on the BD scalar field stated above but not by working with an explicit spacetime solution which was not available at the time. In the present work, however, we investigated closely the known, explicit stationary axisymmetric solution in BD theory. And in particular, when the BDKN solutions can describe non-trivial black hole spacetimes for 
the $\mathrm{BD} \omega$-parametr values $-5 / 2 \leq \omega<-3 / 2$, the accompanying $\mathrm{BD}$ scalar field solution $\Phi(r, \theta)=\Delta^{2 /(2 \omega+3)} \sin ^{4 /(2 \omega+3)} \theta$ turns out not to be a constant field outside the event horizon at $r=r_{+}$. Besides, the energy density of this BD scalar field, $T_{\mu \nu}^{B D} \xi^{\mu} \xi^{\nu}$ whose explicit form was given earlier in eq.(16) does not strictly obey the weak energy condition for all $r$. Namely the value of $T_{\mu \nu}^{B D} \xi^{\mu} \xi^{\nu}$ does not remain non-negative for all $r$. Rather, its value and hence the signature changes from point to point. In short, the Hawking's theorem simply cannot be applied to the present situation and hence the results of the present study needs not be restricted by Hawking's theorem. At this point, it seems appropriate to ask whether the non-trivial BDKN black hole solution studied in the present work can be viewed as a counterexample to the no-hair theorem of black holes. In the loose sense, one may think of the non-trivial behavior of the BD scalar field outside the event horizon as indicating the appearance of "scalar hair". Here, however, we need to be more precise with the nature of no-hair theorem. Following Bizon [11], for instance, a certain theory is said to allow a hairy black hole solution if there is a need to specify quantities other than conserved charges defined at asymptotic infinity such as the mass, angular momentum and the electric charge in order to characterize comletely a stationary black hole solution within that theory. Thus in this stricter sense, the non-trivial BDKN black hole solution studied in the present work does not constitute a hairy black hole solution since both the metric and BD scalar field solutions in eq.(11) are specified completely by the ADM mass $M$, angular momentum per unit mass $a$, and the electric charge $e$ only and no other quantities.

\section{Discussions}

Before we address the physical implication of the non-trivial BDKN black hole solution found in the present work, we would like to comment on a technical issue, i.e., the divergent behavior of the BD scalar field solution on the horizon. And in relation to this, it is interesting to note that our BDKN black hole solution shares two peculiar features, i.e., the divergent behavior of the scalar field on the horizon and the null Hawking radiation, with the well-known Bekenstein black hole solution in Einstein-conformal scalar field theory [12]. Namely, using a suitable solution generation technique, long ago, Bekenstein constructed 
a static, spherically-symmetric black hole solution in which the metric part corresponds to that of extreme Reissner-Nordstrom (RN) black hole and thus represents non-radiating black hole spacetime and the conformal scalar field solution diverges on the horizon. Therefore for direct and parallel comparison between the two solutions, it seems appropriate to take the BDS solution analyzed in detail in the appendix. The two theories, of course, have completely different nature and motivations. The Einstein-conformal scalar field theory has been devised guided mainly by a particular (Weyl) symmetry and the scalar field there is supposed to describe a matter. The BD theory, on the other hand, is an alternative theory to Einstein gravity and the BD scalar field here represents a spacetime-varying effective Newton's constant, not a matter. Thus the divergent behavior of the scalar field in Einstein-conformal scalar field theory could be disastrous but that of the BD scalar field in BD theory essentially represents the vanishing effective Newton's constant in a certain region of spacetime. Besides, since the energy density of the BD scalar field $T_{\mu \nu}^{B D} \xi^{\mu} \xi^{\nu}$ given in eq.(16) vanishes and hence satisfies the weak energy condition on the horizon at which $\Delta=0$ (of course for $-5 / 2 \leq \omega<-3 / 2$ ), we do not worry too much about the divergent behavior of the BD scalar field there. Another interesting contrast is that the metric solution of Bekenstein black hole spacetime there corresponds to a familiar extreme RN metric which is static and spherically-symmetric whereas the BDS metric solution here exhibits a remarkable feature that it is static (i.e., non-rotating) but not spherically-symmetric as we pointed out earlier. Besides, the Bekenstein solution represents a "hairy" black hole [13] whereas our BDS black hole solution carries no hair as we mentioned above. Now, the point of central interest we would like to make is about the issue raised recently by Sudarsky and Zannias [13]. To be a little more concrete, they showed that the divergent behavior of the conformal scalar field solution on the horizon essentially leads the Bekenstein black hole solution to fail to satisfy Einstein field equations particularly on the horizon. They, thus, concluded that the Bekenstein solution cannot be considered as a genuine black hole solution and therefore the black hole no-hair theorem is saved. And as a manifest evidence for their argument against the black hole interpretation, Sudarsky and Zannias demonstrated that by working 
in Eddington-Finkelstein null coordinates, parts of the Einstein field equations can be shown not to hold in a rigorous sense as the left-hand side of the equation, say $R_{\mu \nu}$ vanishes on the horizon (since the metric is that of extreme RN) while the right-hand side, namely the energy-momentum tensor of the conformal scalar field, $8 \pi\left[T_{\mu \nu}-g_{\mu \nu} T_{\lambda}^{\lambda} / 2\right]$ is ill-defined on the horizon as the conformal scalar field diverges there. Thus with this in mind, now we consider the validity of our BDS solution on the horizon. As one can see in the appendix, the 5 non-vanishing components of BD field equations, i.e., $t t, r r, r \theta, \theta \theta$ and $\phi \phi$ parts appear to hold perfectly. In particular, on the horizon and for $-5 / 2 \leq \omega<-3 / 2$, $t$ and $\phi \phi$ parts hold as " $0=0$ ". The other 3 parts, however, hold as " $\infty=\infty$ ". Namely, in these 3 equations, not only the energy-momentum tensor of the BD scalar field on the right-hand side of BD field equations but also the Ricci tensor components on the left-hand side diverges in exactly the same manner on the horizon. Indeed precisely these 3 equations are the ones we need to be careful with. Here, however, we must say that a naive attempt toward the validity check of the BDS solution on the horizon in exactly the same way as Sudarsky and Zannias did for Bekenstein solution seems to be obscured. In fact, the demonstration of Sudarsky and Zannias was successful largely because the simple transformation from spherical to Eddington-Finkelstein null coordinates for the Bekenstein solution was available. Indeed, the virtue of null coordinates is that in terms of which $g_{r r}=0$ and hence $l^{\mu}=(\partial / \partial r)^{\mu}$ becomes a smooth null vector field such that the quantity $R_{\mu \nu} l^{\mu} l^{\nu}$ can be shown to be finite (zero) on the horizon while the right-hand side of Einstein equation, $8 \pi\left[T_{\mu \nu}-g_{\mu \nu} T_{\lambda}^{\lambda} / 2\right] l^{\mu} l^{\nu}$ is ill-defined. In contrast, however, taking a Eddington-Finkelstein-type null coordinates for the BDS solution is not so obvious as in the case of static, spherically-symmetric black hole solutions in Einstein theory and is indeed practically awkward. It is essentially due to the peculiar feature of BDS metric solution which is static but not spherically-symmetric as has been stressed earlier. Thus in the present work, we do not pursue validity check of the BDS solution on the horizon in this direction and leave it as an issue for future investigation. As a result, for the 3 parts of the BD field equations yielding " $\infty=\infty$ ", no definite statement can be made yet concerning whether or not they are valid, i.e., these 3 equations are really 
satisfied on the horizon. Nevertheless, one interesting point we can make is that if the BDS solution does represent a genuine black hole spacetime, then the black hole no-hair theorem appears to survive even in the BD theory of gravity since the BDS (and BDKN as well) spacetime is not a hairy black hole solution as we discussed earlier.

It seems that now the most relevant question to ask is ; if they are genuine, would these non-trivial BDKN black hole spacetimes in BD theory of gravity exhibiting bizzare features such as null radiation really arise in nature? Of course this question needs to be answered very carefully and honestly and the answer for now does not seem to be in the affirmative. Firstly, from field theory's viewpoint, the generic BD theory $\omega$-parameter has to be "positive" in order for the BD scalar field $\Phi$ to have canonical (positive-definite) kinetic energy as can be seen in the BD gravity theory action given in eq.(1). Secondly, it is well-known that the BD gravity theory is in reasonable accord with all available observations and experiments thus far provided $|\omega| \gtrsim 500$ [10]. Since both these constraints on the values of the $\omega$-parameter seem to rule out the range $-5 / 2 \leq \omega<-3 / 2$ in which the BDKN solution could describe non-trivial black hole spacetimes, for now it seems fair to say that these nontrivial BDKN black hole spacetimes different from their general relativistic counterparts are unlikely to arise in nature. This, however, may not be the end of the story. As we have seen in this work, the energy density of the explicit BD scalar field solution (which essentially consists of its kinetic energy) turns out not to satisfy the weak energy condition irrespective of the $\omega$-value. Perhaps this implies that we may abandon the "canonical kinetic energy" condition on the BD scalar field and allow negative- $\omega$ values. Moreover, the lower bound $|\omega| \gtrsim 500$ may be relaxed considerably with the advances in technology associated with astronomical observations and astrophysical experiments. Thus perhaps it might be wise to keep the possibility of non-trivial BDKN black holes alive. As a matter of fact, there is another type of possibility of greater physical significance and relevance. Note that the generic BD $\omega$-parameter is a kind of coupling constant appearing in the BD gravity action. Thus in principle, it should be considered as a "running" coupling constant as a result of renormalization in the quantum gravity context. And its scale-dependent behavior can be 
envisaged as follows. In the BD gravity action given in eq.(1), the term $\sim \omega\left(\nabla_{\alpha} \Phi \nabla^{\alpha} \Phi / \Phi\right)$, like other terms in the action, should be finite. Thus large- $\omega$ indicates the regime where the BD scalar field $\Phi$ remains nearly constant which corresponds to the large-scale present universe limit (in which the BD theory goes over to the general relativity). On the other hand, small- $\omega$ indicates the regime where the BD scalar field varies sizably with space and time which would presumably correspond to the small-scale early universe limit. Thus if we are willing to accept the BD theory as a "better" effective theory of quantum gravity than general relativity to describe the entire stages (scales) of the universe evolution, then at early times when the value of $\omega$ was small such as $-5 / 2 \leq \omega<-3 / 2$, the non-trivial BDKN black holes like the ones studied in this work would have had a chance to form. These "primordial" black holes, unlike their general relativistic counterparts, however, do not evaporate as we discussed earlier. Thus it can be speculated that they might still hide somewhere in the dark side of the space today as a possible constituent of the cold dark matter. After all, new discoveries can be made when we keep our minds as well as eyes open.

\section{Acknowledgements}

This work was supported by grant of Post-doc. Program at Kyungpook National University (1998).

\section{Appendix : Computation of invariant curvature polynomials}

In this appendix, we shall explicitly write down the BD field equations satisfied by the BDS solution given in eq.(13) and then provide the result of the computation of its invariant curvature polynomials such as the Ricci square $R_{\mu \nu} R^{\mu \nu}$ and the Kretschmann curvature invariant $R_{\mu \nu \alpha \beta} R^{\mu \nu \alpha \beta}$. 
First we start with the BD field equations. The tensor part of the vacuum BD field equations that this BDS solution satisfies,

$$
R_{\mu \nu}=\frac{\omega}{\Phi^{2}} \nabla_{\mu} \Phi \nabla_{\nu} \Phi+\frac{1}{\Phi} \nabla_{\mu} \nabla_{\nu} \Phi
$$

has the following 5 non-vanishing components. Here we show that actually they are all satisfied by the BDS solution in the sense that the explicit computation of the left-hand side, i.e., $R_{\mu \nu}$ and the right-hand side, i.e., $\frac{\omega}{\Phi^{2}} \nabla_{\mu} \Phi \nabla_{\nu} \Phi+\frac{1}{\Phi} \nabla_{\mu} \nabla_{\nu} \Phi$ performed with the BDS solution given in eq.(13) precisely agree.

$$
\begin{aligned}
R_{t t} & =\frac{\omega}{\Phi^{2}} \nabla_{t} \Phi \nabla_{t} \Phi+\frac{1}{\Phi} \nabla_{t} \nabla_{t} \Phi \\
& =\frac{1}{r^{4}} \Delta^{-4 /(2 \omega+3)} \sin ^{-8 /(2 \omega+3)} \theta \frac{4}{(2 \omega+3)}\left[\frac{2}{(2 \omega+3)}(r-M)^{2}-M(r-M)+\frac{2}{(2 \omega+3)} \cot ^{2} \theta \Delta\right], \\
R_{r r} & =\frac{\omega}{\Phi^{2}} \nabla_{r} \Phi \nabla_{r} \Phi+\frac{1}{\Phi} \nabla_{r} \nabla_{r} \Phi \\
& =\frac{1}{\Delta^{2}} \frac{4}{(2 \omega+3)}\left[\frac{-4}{(2 \omega+3)}(r-M)^{2}+M(r-M)+\left\{1+\frac{2}{(2 \omega+3)} \cot ^{2} \theta\right\} \Delta\right], \\
R_{r \theta}= & \frac{\omega}{\Phi^{2}} \nabla_{r} \Phi \nabla_{\theta} \Phi+\frac{1}{\Phi} \nabla_{r} \nabla_{\theta} \Phi \\
= & \frac{1}{\Delta} \cot \theta \frac{4}{(2 \omega+3)}\left[\frac{4 \omega}{(2 \omega+3)}(r-M)-(r-2 M)\right], \\
R_{\theta \theta}= & \frac{\omega}{\Phi^{2}} \nabla_{\theta} \Phi \nabla_{\theta} \Phi+\frac{1}{\Phi} \nabla_{\theta} \nabla_{\theta} \Phi \\
= & \frac{1}{\Delta} \frac{4}{(2 \omega+3)}\left[\frac{2}{(2 \omega+3)}(r-M)^{2}+(r-M)(r-2 M)-\left\{1-\left(\frac{2 \omega-1}{2 \omega+3}\right) \cot ^{2} \theta\right\} \Delta\right], \\
R_{\phi \phi}= & \frac{\omega}{\Phi^{2}} \nabla_{\phi} \Phi \nabla_{\phi} \Phi+\frac{1}{\Phi} \nabla_{\phi} \nabla_{\phi} \Phi \\
= & \frac{-1}{\Delta} \Delta^{-4 /(2 \omega+3)} \sin ^{-8 /(2 \omega+3)} \theta \times \\
& \frac{4}{(2 \omega+3)}\left[\left\{\frac{2}{(2 \omega+3)}(r-M)^{2}-(r-M)(r-2 M)\right\} \sin ^{2} \theta-\left(\frac{2 \omega+1}{2 \omega+3}\right) \cos ^{2} \theta \Delta\right] .
\end{aligned}
$$

And the scalar part of the vacuum BD field equations can easily be seen to hold.

Next we turn to the expressions for the invariant curvature polynomials. The Ricci square is calculated to be

$$
\begin{aligned}
& R_{\mu \nu} R^{\mu \nu}=g^{\mu \alpha} g^{\nu \beta} R_{\mu \nu} R_{\alpha \beta} \\
= & \frac{1}{r^{4}} \Delta^{-2(2 \omega+5) /(2 \omega+3)} \sin ^{-8 /(2 \omega+3)} \theta\left[\left\{\frac { 4 } { ( 2 \omega + 3 ) } \left[\frac{2}{(2 \omega+3)}(r-M)^{2}-M(r-M)\right.\right.\right. \\
+ & \left.\left.\frac{2}{(2 \omega+3)} \cot ^{2} \theta \Delta\right]\right\}^{2}
\end{aligned}
$$




$$
\begin{aligned}
& +\left\{\frac{4}{(2 \omega+3)}\left[\frac{-4}{(2 \omega+3)}(r-M)^{2}+M(r-M)+\left(1+\frac{2}{(2 \omega+3)} \cot ^{2} \theta\right) \Delta\right]\right\}^{2} \\
& +\left\{\frac{4}{(2 \omega+3)}\left[\frac{2}{(2 \omega+3)}(r-M)^{2}+(r-M)(r-2 M)-\left(1-\left(\frac{2 \omega-1}{2 \omega+3}\right) \cot ^{2} \theta\right) \Delta\right]\right\}^{2} \\
& \left.+\left\{\frac{4}{(2 \omega+3)}\left[\frac{2}{(2 \omega+3)}(r-M)^{2}-(r-M)(r-2 M)-\left(\frac{2 \omega+1}{2 \omega+3}\right) \cot ^{2} \theta \Delta\right]\right\}^{2}\right] \\
& +\frac{2}{r^{4}} \Delta^{-(2 \omega+7) /(2 \omega+3)} \sin ^{-2(2 \omega+7) /(2 \omega+3)} \theta \cos ^{2} \theta\left[\left\{\frac{4}{(2 \omega+3)}\left[\frac{4 \omega}{(2 \omega+3)}(r-M)-(r-2 M)\right]\right\}^{2}\right] .
\end{aligned}
$$

It is worth noting that this Ricci square vanishes in the limit $\omega \rightarrow \infty$ as it should since in which the BDS solution goes over to the Schwarzschild solution. And the Kretschmann curvature invariant is computed to be

$$
\begin{aligned}
& R_{\mu \nu \alpha \beta} R^{\mu \nu \alpha \beta}=g^{\mu \sigma} g^{\nu \rho} g^{\alpha \lambda} g^{\beta \delta} R_{\nu \alpha \beta}^{\mu} R_{\rho \lambda \delta}^{\sigma} \\
&= \frac{2}{r^{4}} \Delta^{-2(2 \omega+5) /(2 \omega+3)} \sin ^{-8 /(2 \omega+3)} \theta\left[4 \left\{2 \frac{(2 \omega+5)}{(2 \omega+3)^{2}}(r-M)^{2}-\left(\frac{2 \omega+7}{2 \omega+5}\right) M(r-M)+M^{2}\right.\right. \\
&-\left.\frac{1}{(2 \omega+3)}\left[1+\frac{2}{(2 \omega+3)} \cot ^{2} \theta\right] \Delta\right\}^{2} \\
&+ 4\left\{\left[\frac{2}{(2 \omega+3)}(r-M)+(r-2 M)\right]\left[\frac{2}{(2 \omega+3)}(r-M)-M\right]-\frac{1}{(2 \omega+3)}\left[1+\frac{2}{(2 \omega+3)} \cot ^{2} \theta\right] \Delta\right\}^{2} \\
&+\left\{\left[\frac{2}{(2 \omega+3)}(r-M)-(r-2 M)\right]\left[\frac{2}{(2 \omega+3)}(r-M)-M\right]-2 \frac{(2 \omega+1)}{(2 \omega+3)^{2}} \cot ^{2} \theta \Delta\right\}^{2} \\
&+\left.\left\{\left(\frac{2 \omega+7}{2 \omega+3}\right)(r-M)\left[\frac{2}{(2 \omega+3)}(r-M)-(r-2 M)\right]+\left(\frac{2 \omega+1}{2 \omega+3}\right)\left[1+\frac{2}{(2 \omega+3)} \cot ^{2} \theta\right] \Delta\right\}^{2}\right] \\
&+ \frac{2}{r^{4}} \Delta^{-2(2 \omega+5) /(2 \omega+3)} \sin ^{-4(2 \omega+5) /(2 \omega+3)} \theta\left[\left\{\left[\frac{2}{(2 \omega+3)}(r-M)+(r-2 M)\right] \times\right.\right. \\
&+ {\left.\left[\frac{2}{(2 \omega+3)}(r-M)-M\right] \sin ^{2} \theta-\frac{2}{(2 \omega+3)}\left[1+\frac{2}{(2 \omega+3)} \cos ^{2} \theta\right] \Delta\right\}^{2} } \\
&+\left\{\left[\frac{2}{(2 \omega+3)}(r-M)-(r-2 M)\right]\left[\frac{2}{(2 \omega+3)}(r-M)-M\right] \sin ^{2} \theta-2 \frac{(2 \omega+1)}{(2 \omega+3)^{2}} \cos ^{2} \theta \Delta\right\}^{2} \\
&+ 2\left\{(r-M)\left[\frac{2}{(2 \omega+3)}(r-M)+(r-2 M)\right] \sin ^{2} \theta+\left[\frac{2}{(2 \omega+3)}-\left(\frac{2 \omega+5}{2 \omega+3}\right) \sin ^{2} \theta\right] \Delta\right\}^{2} \\
&+\left\{\left(\frac{2 \omega+7}{2 \omega+3}\right)(r-M)\left[\frac{2}{(2 \omega+3)}(r-M)-(r-2 M)\right] \sin ^{2} \theta+\left(\frac{2 \omega+1}{2 \omega+3}\right)\left[1-\left(\frac{2 \omega+5}{2 \omega+3}\right) \cos ^{2} \theta\right] \Delta\right\}^{2} \\
&+\left\{\left[\frac{2}{(2 \omega+3)}(r-M)+(r-2 M)\right]\left[\frac{2}{(2 \omega+3)}(r-M)-M\right] \sin ^{2} \theta\right. \\
&+\left.\frac{2}{(2 \omega+3)}\left[1+\frac{4}{(2 \omega+3)} \cos ^{2} \theta\right] \Delta\right\}^{2} \\
&+\left.\left\{\left[\frac{4}{(2 \omega+3)^{2}}(r-M)^{2}-(r-2 M)^{2}\right] \sin ^{2} \theta+\left(\frac{2 \omega+1}{2 \omega+3}\right)\left[1-\left(\frac{2 \omega-1}{2 \omega+3}\right) \cos ^{2} \theta\right] \Delta\right\}^{2}\right] \\
&+
\end{aligned}
$$




$$
\begin{aligned}
& +\frac{32}{r^{4}} \Delta^{-(2 \omega+7) /(2 \omega+3)} \sin ^{-2(2 \omega+7) /(2 \omega+3)} \theta \cos ^{2} \theta\left[\left\{\frac{1}{(2 \omega+3)}\left[\frac{6}{(2 \omega+3)}(r-M)+(r-4 M)\right]\right\}^{2}\right. \\
& \left.+\left\{\frac{1}{(2 \omega+3)}\left[\frac{4 \omega}{(2 \omega+3)}(r-M)+(r-2 M)\right]\right\}^{2}\right] .
\end{aligned}
$$

Again, it is straightforward to check that this Kretschmann curvature invariant reduces to that of Schwarzschild solution, $48 M^{2} / r^{6}$ in the Einstein gravity limit (i.e., as $\omega \rightarrow \infty$ ) as

it should. In conclusion, a close inspection reveals that $R_{\mu \nu} R^{\mu \nu}$ and $R_{\mu \nu \alpha \beta} R^{\mu \nu \alpha \beta}$ are finite (more precisely vanishes) both on the horizon candidate at which $\Delta=0$ and along the symmetry axis $\theta=0, \pi$ provided the generic $\mathrm{BD} \omega$-parameter takes values in the range $-5 / 2 \leq \omega<-3 / 2$.

\section{References}

[1] C. Brans and C. H. Dicke, Phys. Rev. 124, 925 (1961).

[2] S. Weinberg, Gravitation and Cosmology (Wiley, New York, 1972).

[3] A. I. Janis, D. C. Robinson, and J. Winicour, Phys. Rev. 186, 1729 (1969) ; H. A. Buchdahl, Int. J. Theor. Phys. 6, 407 (1972) ; C. B. G. McIntosh, Commun. Math. Phys. 37, 335 (1974) ; B. O. J. Tupper, Nuovo Cimento 19B, 135 (1974) ; R. N. Tiwari and B. K. Nayak, Phys. Rev. D14, 2502 (1976) ; J. Math. Phys. 18, 289 (1977) ; T. Singh and L. N. Rai, Gen. Rel. Grav. 11, 37 (1979).

[4] R. A. Matzner and C. W. Misner, Phys. Rev. 154, 1229 (1967).

[5] R. M. Misra and D. B. Pandey, J. Math. Phys. 13, 1538 (1972).

[6] R. P. Kerr, Phys. Rev. Lett. 11, 237 (1963) ; E. J. Newman, E. Couch, K. Chinapared, A. Exton, A. Prakash, and R. Torrence, J. Math. Phys. 6, 918 (1965).

[7] R. H. Boyer and R. W. Lindquist, J. Math. Phys. 8, 265 (1967). 
[8] R. Penrose, Lecture at Fifth Texas Symposium on Relativistic Astrophysics, Austin, Texas, December 16, 1970 ; K. S. Thorne and J. J. Dykla, Astrophys. J. L35, 166 (1971).

[9] S. W. Hawking, Commun. Math. Phys. 25, 167 (1972).

[10] See, for example, C. M. Will, Theory and Experiment in Gravitational Physics, revised edition (Cambridge Univ. Press, Cambridge 1993).

[11] P. Bizon, preprint, Jagellonian Univ. Instit. of Phys. Cracow Pol. (1994).

[12] J. D. Bekenstein, Ann. Phys. 82, 535 (1974) ; ibid 91, 72 (1975).

[13] D. Sudarsky and T. Zannias, Phys. Rev. D58, 087502 (1998) ; we thank the referee for drawing our attention to this reference. 